\title{
Desviación traqueal en radiografía de tórax
}

\author{
Jesús Rodríguez Losáñeza , José Juan Carbayo García
}

aResidente de MFyC. Centro de Salud Talavera Centro. Talavera de la Reina. Toledo. España.

'Médico de Familia. Centro de Salud Talavera Centro.Talavera de la Reina. Toledo. España.

Correspondencia: José Juan Carbayo García, Centro de Salud Talavera Centro,

C/ José Luis Gallo 2, 45600. Talavera de la Reina. Toledo, Teléfono: 925800950, Correo electrónico: jjcarbayo@sescam.jccm.es.

Recibido el 28 de febrero de 2012.

Aceptado para su publicación el 2 de abril de 2012.

\section{RESUMEN}

Paciente varón de 67 años de edad que acude a consulta de atención primaria con sintomatología de astenia, anorexia, progresiva pérdida de peso, así como disnea de medianos esfuerzos. No refiere síntomas aparentes de insuficiencia cardiaca, no presenta disfagia ni disfonía y no tiene fiebre.

En la Radiografía simple de tórax se observa una compresión y desviación de la columna de aire traqueal hacia el lado derecho y discreta hiperinsuflación de ambos campos pulmonares.

Se realiza Ecografía en el Centro de Salud apreciando un bocio intratorácico, no se encuentran otras alteraciones en mediastino.

Palabras clave. Desviación traqueal, Tiroides, Bocio multinodular intratorácico.

\section{ABSTRACT}

Tracheal curvature in chest X-ray

A 67 year old male patient sought medical attention at a primary health centre with symptoms of asthenia, anorexia, progressive weight loss, along with dyspnea upon slight exertion. He did not show symptoms of heart failure, did not present dysphagia or dysphonia and did not have fever. In a simple chest X-ray, compression and right curvature of the tracheal air column and discreet overinflation of both lung fields could be seen. An ultrasound scan was carried out at the health centre and intrathoracic goitre was visible. No other alterations in the mediastinum were found. Key words. Tracheal deviatión, Thyroid, multinodular intrathoracic Goiter.

\section{INTRODUCCIÓN}

En la valoración de una Radiografía de tórax debemos prestar atención al mediastino. Si aparece ensanchado en la parte superior, debemos pensar en hipertrofia tiroidea, tumores tímicos o arteria inominada. Si es en la parte central o inferior, pensaremos en dilatación aórtica, adenomegalias, dilatación de esófago o hernia de hiato'1.

Un tiroides hipertrófico comprime o desplaza la tráquea (algo que no sucede con la arteria inominada tortuosa), tiene un margen bien definido y no altera el borde blanco paratraqueal como ocurre en presencia de otras masas paratraqueales ${ }^{1}$.

La Ecografía resulta de gran utilidad en Atención Primaria. A nivel cervical, en el diagnóstico del bocio, proporcionando información tanto sobre su tamaño como sobre las características de la glándula y a nivel abdominal, al permitirnos valorar entre otras estructuras, aorta y mediastino.

El bocio es una patología que se caracteriza por el crecimiento lento y progresivo de la glándula tiroides. Por lo general, la mayoría de los bocios son pequeños y pueden pasar desapercibidos, sin embargo, hay situaciones en las que tiende a convertirse en multinodular, con funcionamiento propio y a situarse a nivel retroesternal, comprometiendo estructuras vecinas como la 
tráquea y esófago, siendo su manifestación clínica más importante la disnea².

En determinados bocios puede haber una compresión y/o desplazamiento traqueal severo. En estos casos, cualquier cambio brusco tiroideo (hemorragia intrabocio, etc.) puede desencadenar un cuadro respiratorio agudo e incluso ocasionar el fallecimiento del paciente por asfixia ${ }^{3}$

\section{OBSERVACIONES CLÍNICAS}

Varón de 67 años de edad, fumador activo de 52 paquetes-año, con antecedentes personales de Dislipemia, Hipertensión Ocular, Deterioro Cognitivo Leve de origen vascular y Síndrome depresivo crónico.

Medicación actual: Escitalopram 10 mg, Risperidona $1 \mathrm{mg}$, Simvastatina $20 \mathrm{mg}$. Acido Acetilsalicílico 100 mg, Cosopt ${ }^{\mathrm{R}}$ Colirio.

Acude a la consulta de atención primaria con sintomatología de astenia, anorexia, progresiva pérdida de peso, así como disnea de medianos esfuerzos. No refiere síntomas aparentes de insuficiencia cardiaca, de disfagia o disfonía y no presenta fiebre.

Se solicita Radiografía de tórax (se trata de una prueba fácil, rápida, barata, que aporta una gran cantidad de información para el diagnóstico de las enfermedades respiratorias y de amplio uso en atención primaria) y Espirometría para valorar la situación funcional. En la Radiografía simple

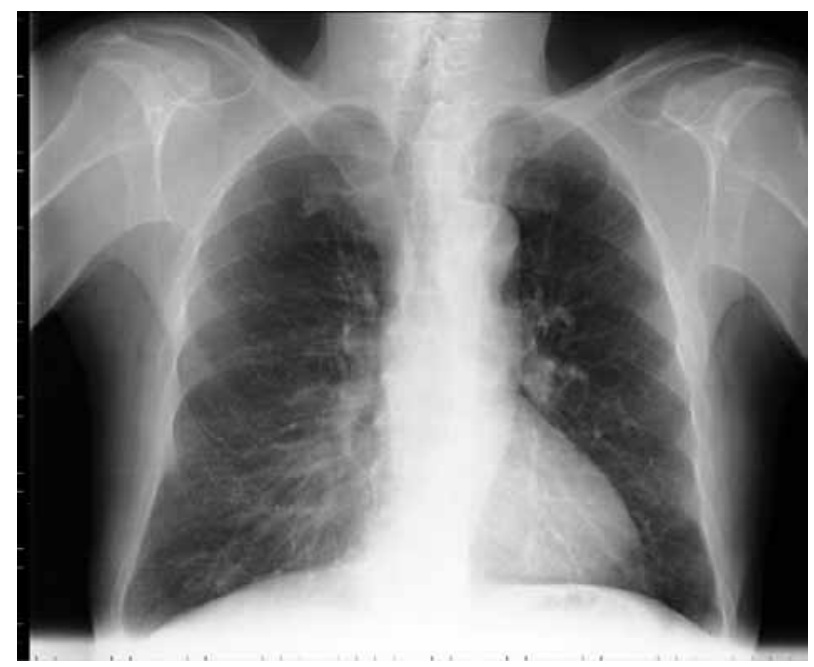

cervicotorácica posteroanterior (Fig.1) se observa una compresión y desviación de la columna de aire traqueal hacia el lado derecho respecto a la línea media que forman las apófisis espinosas de la columna cervicotorácica. Los pulmones aparecen discretamente radiotransparentes, hiperinsuflados con diafragmas aplanados.

Los resultados de la espirometría muestran un patrón restrictivo leve (FVC: 73\%, FEV1: 69\%, FEV1/FVC: 97\%).

La Ecografía resulta de gran utilidad en Atención Primaria, nos permite ver elementos normales y patológicos del cuerpo humano sin apenas efectos negativos. A nivel cervical nos facilita el diagnóstico del bocio, proporcionando información tanto sobre su tamaño como sobre las características de la glándula. A nivel abdominal nos permite valorar entre otras estructuras, aorta y mediastino.

En el paciente, a nivel tiroideo apreciamos un lóbulo izquierdo muy aumentado de tamaño que se introduce en tórax impidiendo su correcta medición, con múltiples nódulos, zonas hipoecogénicas y alteración de la ecoestructura (Fig. 2-3).

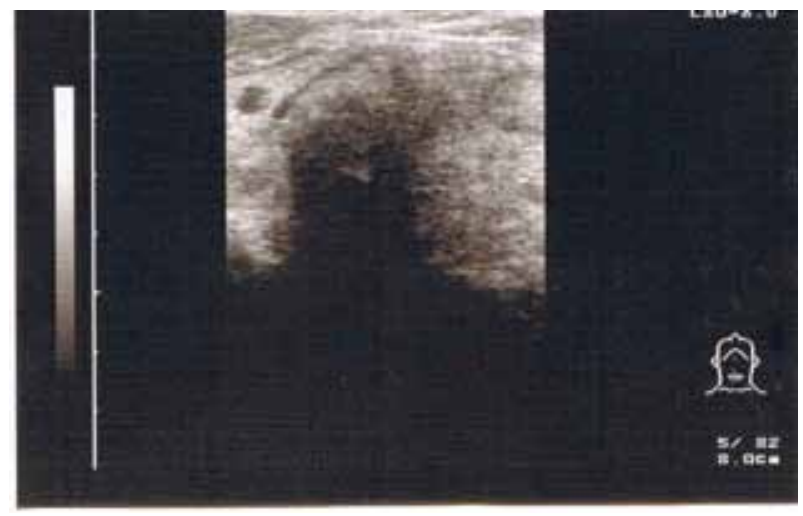

Fig. 2. Corte transversal a nivel tiroideo. Lóbulo izqdo. muy aumentado de tamaño, con múltiples nódulos y alteración de la ecoestructura.

Figura 1. Radiografía Postero-Anterior de Tórax 


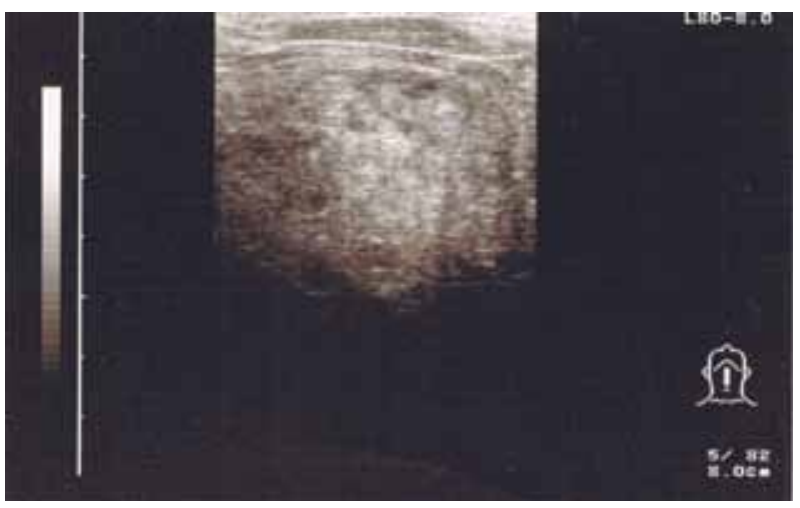

Fig. 3. Corte longitudinal a nivel del Lóbulo Izqdo. Tiroideo.

\section{COMENTARIO}

Por bocio multinodular se entiende el aumento irregular de tamaño de la glándula tiroides secundario al estímulo que supone una elevación de la $\mathrm{TSH}^{4}$. Entre sus formas de presentación destaca el síntoma compresivo ${ }^{5}$, como sucede en el paciente del caso, que se produce sobre todo cuando el bocio multinodular crece hacia el tórax y crea un conflicto de espacio a nivel del estrecho torácico superior. Pueden darse problemas respiratorios que supongan una amenaza grave para la vida del paciente, pero que en nuestro caso no impresionan de gravedad y que pudieran precipitarse debido a una infección respiratoria o por cambios en la posición del cuello.

Los bocios asociados con graves problemas respiratorios tienen mayor riesgo de albergar un car- cinoma ${ }^{6}$, bien es cierto que el bocio multinodular no parece ser un factor de riesgo para el desarrollo del cáncer de tiroides, pero éste, ciertamente, puede desarrollarse sobre un bocio multinodular.

El diagnóstico precoz del bocio y su adecuado control y seguimiento ayuda a prevenir posibles comorbilidades asociadas como la compresión de la vía aérea.

La combinación de técnicas diagnósticas disponibles en AP consigue un correcto diagnóstico y evita derivaciones innecesarias o mal enfocadas que encarecen y enlentecen el proceso.

\section{BIBLIOGRAFÍA}

1. Corne J, Carroll M, Broown I, Delany D. Consulta rápida en radiología Torácica. $2^{\mathrm{a}} \mathrm{ed}$. Madrid: Elsevier. 2006.

2. Hurley DL, Gharib H. Evaluation and management of multinodular goiter. Otolaryngol Clin North Am 1996; 29: 527-540.

3. Singh B, Lucente FE, Shaha AR. Substernal goiter: a clinical review. Am J Otolaryngol 1994;15:409-16.

4. Allo MD, Thompson NW. Rationale for the operative management of substernal goiter. Surgery 1983; 94: 969-977.

5. Bahn RS, Castro MR. Approach to the patient with nontoxic multinodular goiter. J Clin Endocrinol Metab. 2011 May;96(5):1202-12.

6. Ermans AM: Endemic goiter. In Ingbar SH, Braverman LE (eds). Werner's The Tryroid- Afundamental and clinical text. Philadelphia, PA. Lippincott, 186, 705721. 